\title{
A STUDY OF POSITIVE PERIODIC SOLUTIONS FOR SECOND-ORDER DELAYED DIFFERENTIAL EQUATIONS
}

\section{YUE LIANG and DI ZHANG}

Science College of Gansu

Agricultural University

Lanzhou 730070

P. R. China

e-mail: liangyuegsau@163.com

aqsy0008@163.com

\begin{abstract}
This paper deals with the existence of positive $\delta$-periodic solutions for secondorder differential equation with delay of the form

$$
-x^{\prime \prime}(t)+M x(t)=f(t, x(t), x(t-\tau)), \quad t \in \mathbb{R},
$$

where $M>0$ is a constant, $f: \mathbb{R} \times \mathbb{R}^{+} \times \mathbb{R}^{+} \rightarrow \mathbb{R}^{+}$is a continuous function, $f(t, u, v)$ is $\delta$-periodic in $t, \tau>0$ is a constant which denotes the time delay. We first establish the positivity estimation for the linear equation. Then two existence theorems of positive $\delta$-periodic solutions are obtained when $f$ satisfies some inequality conditions. The discussion is based on the fixed point index theory in cones.
\end{abstract}

2010 Mathematics Subject Classification: 34C25, 47H10.

Keywords and phrases: second-order differential equation, positive $\delta$-periodic solution, fixed point index, delay, existence.

Received February 27, 2018; Revised March 21, 2018

(C) 2018 Scientific Advances Publishers 


\section{Introduction}

In this paper, we study the existence of positive $\delta$-periodic solutions for second-order differential equation with delay of the form

$$
-x^{\prime \prime}(t)+M x(t)=f(t, x(t), x(t-\tau)), \quad t \in \mathbb{R},
$$

where $M>0$ is a constant, $f: \mathbb{R} \times \mathbb{R}^{+} \times \mathbb{R}^{+} \rightarrow \mathbb{R}^{+}$is a continuous function, $f(t, u, v)$ is $\delta$-periodic in $t, \tau>0$ is a constant.

Since the differential equation with delay has extensive physical, biological and engineering background, it has been an important branch of differential equation theory. The existence of periodic solutions for delayed differential equation has been studied in recent years and an increasing number of papers emerged, see $[3,6,7,10,11]$ and the reference therein. But the positive periodic solutions are more important in applications. The existence of positive periodic solutions of differential equations without delay has been researched, see [4, 5]. Recently, the authors in $[6,8,9]$ investigated the existence of positive periodic solutions for delayed differential equations. Motivated by these papers, we consider the existence of positive $\delta$-periodic solutions of Equation (1.1). We first establish the positivity estimation of the linear equation corresponding to Equation (1.1), then by using fixed point index in cones, two existence theorems of positive $\delta$-periodic solutions for Equation (1.1) are proved when nonlinearity $f$ satisfies inequality conditions.

\section{Preliminaries}

Let $\delta>0$ be a constant. The set of all continuous $\delta$-periodic function $x(t)$ will be denoted by $C_{\delta}(\mathbb{R})$. Then $C_{\delta}(\mathbb{R})$ is a Banach space with norm $\|x\|_{C}=\max _{t \in[0, \delta]}|u(t)|$. The cone of all nonnegative functions in $C_{\delta}(\mathbb{R})$ will be denoted by $C_{\delta}^{+}(\mathbb{R})$. 
For $g \in C_{\delta}(\mathbb{R})$, we first consider the linear differential equation

$$
-x^{\prime \prime}(t)+M x(t)=g(t), \quad t \in \mathbb{R} .
$$

Let $M>0$ and $\eta=\sqrt{M}$, from Lemma 2.1 of [5], the Equation (2.1) has a unique $\delta$-periodic solution $u(t)$ which is expressed by

$$
x(t)=\int_{t-\delta}^{t} \Phi(t-s) g(s) d s:=(\Psi g)(t), \quad t \in \mathbb{R},
$$

where

$$
\Phi(t)=\frac{\cosh \eta(t-\delta / 2)}{2 \eta \sinh (\eta \delta / 2)}, \quad t \in[0, \delta]
$$

and $\Psi: C_{\delta}(\mathbb{R}) \rightarrow C_{\delta}(\mathbb{R})$ is a completely continuous linear operator.

By (2.2) and (2.3), we can obtain

$$
\|\Psi\|=\int_{0}^{\delta} \Phi(s) d s=\frac{1}{M},
$$

and

$$
\frac{1}{2 \eta \sinh (\eta \delta / 2)} \leq \Phi(t) \leq \frac{\cosh (\eta \delta / 2)}{2 \eta \sinh (\eta \delta / 2)}
$$

For the sake of brevity, let $\kappa=(\cosh (\eta \delta / 2))^{-1}$. Then $\kappa>0$.

For any $g \in C_{\delta}(\mathbb{R})$, we study the second-order linear delayed differential equation

$$
-x^{\prime \prime}(t)+M x(t)+N x(t-\tau)=g(t), \quad t \in \mathbb{R},
$$

where $M>0$ and $N \geq 0$. From [7], we have the following result:

Lemma 1. For any $g \in C_{\delta}(\mathbb{R})$, if $0 \leq N<\kappa^{2} M$, then the linear delayed differential equation (2.6) has a unique $\delta$-periodic solution $x(t)$ which is given by

$$
x(t)=\left(I+\Psi \circ B_{\tau}\right)^{-1} \int_{t-\delta}^{t} \Phi(t-s) g(s) d s, \quad t \in \mathbb{R},
$$


where $B_{\tau}: C_{\delta}(\mathbb{R}) \rightarrow C_{\delta}(\mathbb{R})$ is defined by

$$
B_{\tau} x(t)=N x(t-\tau), \quad t \in \mathbb{R}, \tau>0 .
$$

Let $G(x)(t):=f(t, x(t), x(t-\tau))+N x(t-\tau)$. An operator $\Lambda: C_{\delta}(\mathbb{R}) \rightarrow$ $C_{\delta}(\mathbb{R})$ is define by

$$
(\Lambda x)(t)=\left(I+\Psi \circ B_{\tau}\right)^{-1} \int_{t-\delta}^{t} \Phi(t-s) G(x)(s) d s, \quad t \in \mathbb{R} .
$$

Then by Lemma $1, \Lambda: C_{\delta}(\mathbb{R}) \rightarrow C_{\delta}(\mathbb{R})$ is continuous and the $\delta$-periodic solution of the second-order delayed differential equation (1.1) is equivalent to the fixed point of $\Lambda$.

Choose a subcone of $C_{\delta}^{+}(\mathbb{R})$ as

$$
\Pi=\left\{x \in C_{\delta}^{+}(\mathbb{R}): x(t) \geq \kappa x(s), \forall t, s \in \mathbb{R}\right\}
$$

By Lemma 1, we obtain the following lemma:

Lemma 2. $\Lambda: \Pi \rightarrow \Pi$ is completely continuous.

Proof. By the complete continuity of $\Psi$ and the definition of $\Lambda$, it is easy to see that $\Lambda: \Pi \rightarrow C_{\delta}(\mathbb{R})$ is completely continuous. It remains to prove $\Lambda(\Pi) \subset \Pi$.

For any $x \in \Pi$, it follows from the definition of $\Lambda$ that

$$
\left(I+\Psi \circ B_{\tau}\right)(\Lambda x)(t)=\int_{t-\delta}^{t} \Phi(t-s) G(x)(s) d s, \quad t \in \mathbb{R} .
$$

From (2.5) and (2.9), for any $t, s \in \mathbb{R}$, we have

$$
\begin{aligned}
\left(I+\Psi \circ B_{\tau}\right)(\Lambda x)(s) & \leq \frac{\cosh (\eta \delta / 2)}{2 \eta \sinh (\eta \delta / 2)} \int_{s-\delta}^{s} G(x)(s) d s \\
& =\frac{\cosh (\eta \delta / 2)}{2 \eta \sinh (\eta \delta / 2)} \int_{0}^{\delta} G(x)(s) d s
\end{aligned}
$$


and

$$
\begin{aligned}
\left(I+\Psi \circ B_{\tau}\right)(\Lambda x)(t) & \geq \frac{1}{2 \eta \sinh (\eta \delta / 2)} \int_{t-\delta}^{t} G(x)(s) d s \\
& =\frac{1}{2 \eta \sinh (\eta \delta / 2)} \int_{0}^{\delta} G(x)(s) d s .
\end{aligned}
$$

This implies that

$$
\left(I+\Psi \circ B_{\tau}\right)(\Lambda x)(t) \geq \kappa\left(I+\Psi \circ B_{\tau}\right)(\Lambda x)(s), \quad \forall t, s \in \mathbb{R} .
$$

From the proof of Lemma 2.1 in [7], it follows that $\left(I+\Psi \circ B_{\tau}\right)^{-1}$ is a positive operator. Hence by (2.10), we have

$$
(\Lambda x)(t) \geq \kappa(\Lambda x)(s), \quad \forall t, s \in \mathbb{R} .
$$

Hence, $\Lambda: \Pi \rightarrow \Pi$ is completely continuous.

Next, we recall some conclusions of fixed point index in cones. Let $\Xi$ be a Banach space and $\Delta \subset \Xi$ a closed convex cone in $\Xi$. Assume that $\Theta$ is a bounded open subset of $\Xi$ with boundary $\partial \Theta$, and $\Delta \cap \Theta$ is nonempty. Let $\Lambda: \Delta \cap \bar{\Theta} \rightarrow \Delta$ be a completely continuous mapping. If $\Lambda u \neq u$ for any $u \in \Delta \cap \partial \Theta$, then the fixed point index $i(\Lambda, \Delta \cap \Theta, \Delta)$ is well defined. If $i(\Lambda, \Delta \cap \Theta, \Delta) \neq 0$, then $\Lambda$ has a fixed point in $\Delta \cap \Theta$. For more details of the concepts and conclusions on the fixed point index, we refer to $[1,2]$. The following two lemmas are needed in our arguments.

Lemma 3. Let $\Theta$ be a bounded open subset of $\Xi$ with $\theta \in \Theta$ and $\Lambda: \Delta \cap \bar{\Theta} \rightarrow \Delta$ be a completely continuous mapping. If

$$
\varrho \Lambda x \neq x, \quad \forall x \in \Delta \cap \partial \Theta, \quad 0<\varrho \leq 1,
$$

then $i(\Lambda, \Delta \cap \Theta, \Delta)=1$. 
Lemma 4. Let $\Theta$ be a bounded open subset of $\Xi$ and $\Lambda: \Delta \cap \bar{\Theta} \rightarrow \Delta$ be a completely continuous mapping. If there exists an $e \in \Delta \backslash\{\theta\}$ such that

$$
x-\Lambda x \neq \nu e, \quad \forall x \in \Delta \bigcap \partial \Theta, \quad \nu \geq 0,
$$

then $i(\Lambda, \Delta \cap \Theta, \Delta)=0$.

\section{Main Results}

Theorem 1. Let $f: \mathbb{R} \times \mathbb{R}^{+} \times \mathbb{R}^{+} \rightarrow \mathbb{R}^{+}$be continuous and $f(t, u, v)$ $\delta$-periodic in $t$ for every $u, v \in \mathbb{R}^{+}$. If $M>\kappa^{-2} N \geq 0$ and $f$ satisfies the following conditions:

(H1) There exist two positive constants $\alpha_{1}$ and $\alpha_{2}$ satisfying $\alpha_{1}+\alpha_{2}<M$ and $\varepsilon>0$ such that

$$
f(t, u, v) \leq \alpha_{1} u+\alpha_{2} v
$$

for any $t \in \mathbb{R}$ and $u, v \in[0, \varepsilon]$.

(H2) There exist two positive constants $\beta_{1}$ and $\beta_{2}$ satisfying $\beta_{1}+\beta_{2}>M$ and $h_{0} \in C_{\delta}^{+}(\mathbb{R})$ such that

$$
f(t, u, v) \geq \beta_{1} u+\beta_{2} v-h_{0}(t)
$$

for any $t \in \mathbb{R}$ and $u, v \in \mathbb{R}^{+}$,

then the Equation (1.1) has at least one positive $\delta$-periodic solution.

Proof. Define an operator $\Lambda: C_{\delta}(\mathbb{R}) \rightarrow C_{\delta}(\mathbb{R})$ as in (2.8). By Lemma 2, $\Lambda: \Pi \rightarrow \Pi$ is completely continuous. Let

$$
\Delta_{r}=\left\{x \in C_{\delta}(\mathbb{R}):\|x\|_{C}<r\right\}, \quad \Delta_{R}=\left\{x \in C_{\delta}(\mathbb{R}):\|x\|_{C}<R\right\},
$$

where $0<r<R<+\infty$. Then $\Delta_{r}$ and $\Delta_{R}$ are bounded open subsets of $C_{\delta}(\mathbb{R})$. We will show that $\Lambda$ has a fixed point in $\Pi \cap\left(\Delta_{R} \backslash \bar{\Delta}_{r}\right)$ for some $r>0$ and $R>0$. 
Let $r \in(0, \varepsilon)$, where $\varepsilon$ is the positive constant in condition (H1). We prove that $\Lambda$ satisfies the condition of Lemma 3 in $\Pi \cap \Delta_{r}$. If there exist $x_{0} \in \Pi \cap \partial \Delta_{r}$ and $0<\varrho_{0} \leq 1$ such that

$$
\varrho_{0} \Lambda x_{0}=x_{0},
$$

by the definition of $\Lambda$ and Lemma 1, we have

$$
-x_{0}^{\prime \prime}(t)+M x_{0}(t)+N x_{0}(t-\tau)=\varrho_{0} f\left(t, x_{0}(t), x_{0}(t-\tau)\right)+\varrho_{0} N x_{0}(t-\tau), t \in \mathbb{R} .
$$

Since $0<\varrho_{0} \leq 1$, deduced from the above inequality that

$$
-x_{0}^{\prime \prime}(t)+M x_{0}(t) \leq f\left(t, x_{0}(t), x_{0}(t-\tau)\right), \quad t \in \mathbb{R} .
$$

By $x_{0} \in \Pi \cap \partial \Delta_{r}$, we have

$$
0 \leq x_{0}(t) \leq\left\|x_{0}\right\|_{C}=r<\varepsilon,
$$

and

$$
0 \leq x_{0}(t-\tau) \leq\left\|x_{0}\right\|_{C}=r<\varepsilon .
$$

Hence, by condition (H1), we can obtain

$$
f\left(t, x_{0}(t), x_{0}(t-\tau)\right) \leq \alpha_{1} x_{0}(t)+\alpha_{2} x_{0}(t-\tau), \quad t \in \mathbb{R} .
$$

Combining this inequality with (3.2), we have

$$
-x_{0}^{\prime \prime}(t)+M x_{0}(t) \leq \alpha_{1} x_{0}(t)+\alpha_{2} x_{0}(t-\tau), \quad t \in \mathbb{R} .
$$

Integrating both sides of this inequality from 0 to $\delta$ and using the periodicity of $x_{0}$, we have

$$
\begin{aligned}
M \int_{0}^{\delta} x_{0}(t) d t & \leq \alpha_{1} \int_{0}^{\delta} x_{0}(t) d t+\alpha_{2} \int_{0}^{\delta} x_{0}(t-\tau) d t \\
& =\left(\alpha_{1}+\alpha_{2}\right) \int_{0}^{\delta} x_{0}(t) d t .
\end{aligned}
$$


Since $x_{0} \in \Pi$, by the definition of $\Pi$, we have

$$
x_{0}(t) \geq \kappa x_{0}(s), \quad \forall t, s \in \mathbb{R} .
$$

Hence, we can obtain

$$
\left(M-\alpha_{1}-\alpha_{2}\right) \kappa \delta x_{0}(s) \leq\left(M-\alpha_{1}-\alpha_{2}\right) \int_{0}^{\delta} x_{0}(t) d t \leq 0, \quad \forall s \in \mathbb{R} .
$$

Since $M>\alpha_{1}+\alpha_{2}, \kappa>0$ and $\delta>0$, it follows that $x_{0}(s) \leq 0$ for any $s \in \mathbb{R}$, which contracts with $x_{0} \in \Pi \cap \partial \Delta_{r}$. This means that $\Lambda$ satisfies the condition of Lemma 3. By Lemma 3,

$$
i\left(\Lambda, \Pi \cap \Delta_{r} \Pi\right)=1 .
$$

On the other hand, we show that $\Lambda$ satisfies the condition of Lemma 4 in $\Pi \cap \Delta_{R}$. Let $e(t) \equiv 1$ for any $t \in \mathbb{R}$. Then $e \in \Pi \backslash\{\theta\}$. We show that if $R$ is large enough, then $x-\Lambda x \neq v e$ for any $x \in \Pi \cap \partial \Delta_{R}$ and $v \geq 0$. In fact, if there exist $x_{1} \in \Pi \cap \partial \Delta_{R}$ and $\nu_{1} \geq 0$ such that

$$
x_{1}-\Lambda x_{1}=v_{1} e
$$

namely, $x_{1}-v_{1} e=\Lambda x_{1}$. By the definition of $\Lambda$, we have

$$
-x_{1}^{\prime \prime}(t)+M x_{1}(t)-v_{1}(M+N)=f\left(t, x_{1}(t), x_{1}(t-\tau)\right), \quad t \in \mathbb{R} .
$$

By condition (H2), it follows that

$$
-x_{1}^{\prime \prime}(t)+M x_{1}(t) \geq \beta_{1} x_{1}(t)+\beta_{2} x_{1}(t-\tau)-h_{0}(t), \quad t \in \mathbb{R} .
$$

Integrating both sides of this inequality from 0 to $\delta$ and using the periodicity of $x_{1}$, we have

$$
\begin{aligned}
M \int_{0}^{\delta} x_{1}(t) d t & \geq \beta_{1} \int_{0}^{\delta} x_{1}(t) d t+\beta_{2} \int_{0}^{\delta} x_{1}(t-\tau) d t-\int_{0}^{\delta} h_{0}(t) d t \\
& =\left(\beta_{1}+\beta_{2}\right) \int_{0}^{\delta} x_{1}(t) d t-\int_{0}^{\delta} h_{0}(t) d t .
\end{aligned}
$$


This implies that

$$
\left(\beta_{1}+\beta_{2}-M\right) \int_{0}^{\delta} x_{1}(t) d t \leq \int_{0}^{\delta} h_{0}(t) d t \leq \delta\left\|h_{0}\right\|_{C}
$$

Since $x_{1} \in \Pi, x_{1}(t) \geq \kappa x_{1}(s)$ for any $t, s \in \mathbb{R}$. So,

$$
\left(\beta_{1}+\beta_{2}-M\right) \kappa \delta x_{1}(s) \leq \delta\left\|h_{0}\right\|_{C}, \quad \forall s \in \mathbb{R} .
$$

Hence, we can obtain

$$
\left\|x_{1}\right\|_{C} \leq \frac{\left\|h_{0}\right\|_{C}}{\left(\beta_{1}+\beta_{2}-M\right) \kappa}
$$

Let $R>\max \left\{\frac{\left\|h_{0}\right\|_{C}}{\left(\beta_{1}+\beta_{2}-M\right) \kappa}, r\right\}$. Then the condition of Lemma 4 holds.

By Lemma 4, we have

$$
i\left(\Lambda, \Pi \cap \Delta_{R}, \Pi\right)=0 .
$$

Combining (3.3) and (3.4), using the additivity of fixed point index, we have

$$
i\left(\Lambda, \Pi \cap\left(\Delta_{R} \backslash \bar{\Delta}_{r}\right), \Pi\right)=i\left(\Lambda, \Pi \cap \Delta_{R}, \Pi\right)-i\left(\Lambda, \Pi \cap \Delta_{r}, \Pi\right)=-1 \neq 0 .
$$

Hence, $\Lambda$ has at least one fixed point in $\Pi \cap\left(\Delta_{R} \backslash \bar{\Delta}_{r}\right)$, which is a positive $\delta$-periodic solution of the second-order delayed differential equation (1.1).

Theorem 2. Let $f: \mathbb{R} \times \mathbb{R}^{+} \times \mathbb{R}^{+} \rightarrow \mathbb{R}^{+}$be continuous and $f(t, u, v)$ $\delta$-periodic in $t$ for every $u, v \in \mathbb{R}^{+}$. If $M>\kappa^{-2} N \geq 0$ and $f$ satisfies the following conditions:

(H3) There exist two positive constants $\beta_{1}$ and $\beta_{2}$ satisfying $\beta_{1}+\beta_{2}>M$ and $\varepsilon>0$ such that

$$
f(t, u, v) \geq \beta_{1} u+\beta_{2} v,
$$

for any $t \in \mathbb{R}$ and $u, v \in[0, \varepsilon]$. 
(H4) There exist two positive constants $\alpha_{1}$ and $\alpha_{2}$ satisfying $\alpha_{1}+\alpha_{2}<M$ and $h_{1} \in C_{\delta}^{+}(\mathbb{R})$ such that

$$
f(t, u, v) \leq \alpha_{1} u+\alpha_{2} v+h_{1}(t),
$$

for any $t \in \mathbb{R}$ and $u, v \in \mathbb{R}^{+}$,

then the Equation (1.1) has at least one positive $\delta$-periodic solution.

Proof. Define an operator $\Lambda: C_{\delta}(\mathbb{R}) \rightarrow C_{\delta}(\mathbb{R})$ as in (2.8). Then $\Lambda: \Pi \rightarrow \Pi$ is completely continuous. Let $\Delta_{r}$ and $\Delta_{R}$ be defined by (3.1) for any $0<r<R<+\infty$. We show that $\Lambda$ has a fixed point in $\Pi \cap\left(\Delta_{R} \backslash \bar{\Delta}_{r}\right)$ for some positive numbers $r$ and $R$.

Let $r \in(0, \varepsilon)$, where $\varepsilon$ is the positive number in condition (H3). Choose $e(t) \equiv 1$ for any $t \in \mathbb{R}$. Then $e \in \Pi \backslash\{\theta\}$. We show that $\Lambda$ satisfies the condition of Lemma 4 in $\Pi \cap \Delta_{r}$. In fact, if there exist $x_{0} \in \Pi \cap \partial \Delta_{r}$ and $\nu_{0} \geq 0$ such that

$$
x_{0}-v_{0} e=\Lambda x_{0}
$$

then by the definition of $\Lambda$, we can obtain

$$
-x_{0}^{\prime \prime}(t)+M x_{0}(t) \geq f\left(t, x_{0}(t), x_{0}(t-\tau)\right), \quad t \in \mathbb{R} .
$$

Since $x_{0} \in \Pi \cap \partial \Delta_{r}$, it follows that $x_{0}(t), x_{0}(t-\tau) \in[0, \varepsilon]$. By the condition (H3), we have

$$
f\left(t, x_{0}(t), x_{0}(t-\tau)\right) \geq \beta_{1} x_{0}(t)+\beta_{2} x_{0}(t-\tau), \quad t \in R .
$$

Combining this inequality with (3.5), we have

$$
-x_{0}^{\prime \prime}(t)+M x_{0}(t) \geq \beta_{1} x_{0}(t)+\beta_{2} x_{0}(t-\tau), \quad t \in R .
$$


Integrating both sides of this inequality from 0 to $\delta$ and using the periodicity of $x_{0}$, we have

$$
\begin{aligned}
M \int_{0}^{\delta} x_{0}(t) d t & \geq \beta_{1} \int_{0}^{\delta} x_{1}(t) d t+\beta_{2} \int_{0}^{\delta} x_{0}(t-\tau) d t \\
& =\left(\beta_{1}+\beta_{2}\right) \int_{0}^{\delta} x_{0}(t) d t
\end{aligned}
$$

This implies that

$$
\left(\beta_{1}+\beta_{2}-M\right) \int_{0}^{\delta} x_{0}(t) d t \leq 0
$$

Since $x_{0} \in \prod$, it follows that

$$
\left(\beta_{1}+\beta_{2}-M\right) \kappa \delta x_{0}(s) \leq 0, \quad \forall s \in \mathbb{R}
$$

This implies $x_{0}(t) \leq 0$ for any $t \in \mathbb{R}$ because of $\beta_{1}+\beta_{2}>M, \kappa>0$ and $\delta>0$, which contracts with $x_{0} \in \Pi \cap \partial \Delta_{r}$. Hence, by Lemma 4, we have

$$
i\left(\Lambda, \Pi \cap \Delta_{r}, \Pi\right)=0 .
$$

On the other hand, we show that $\Lambda$ satisfies the condition of Lemma 3 in $\Pi \cap \Delta_{R}$ when $R$ is large enough. In face, if there exist $x_{1} \in \Pi \cap \partial \Delta_{R}$ and $0<\varrho_{1} \leq 1$ such that

$$
x_{1}=\varrho_{1} \Lambda x_{1} .
$$

By the definition of $\Lambda$, we have

$$
-x_{1}^{\prime \prime}(t)+M x_{1}(t) \leq f\left(t, x_{1}(t), x_{1}(t-\tau)\right), \quad t \in R .
$$

Since $x_{1} \in \Pi \cap \partial \Delta_{r}$, by the condition (H4), we have

$$
-x_{1}^{\prime \prime}(t)+M x_{1}(t) \leq \alpha_{1} x_{1}(t)+\alpha_{2} x_{1}(t-\tau)+h_{1}(t), \quad t \in R .
$$


Integrating both sides of this inequality from 0 to $\delta$ and using the periodicity of $x_{1}$, we have

$$
\begin{aligned}
M \int_{0}^{\delta} x_{1}(t) d t & \leq \alpha_{1} \int_{0}^{\delta} x_{1}(t) d t+\alpha_{2} \int_{0}^{\delta} x_{0}(t-\tau) d t+\int_{0}^{\delta} h_{1}(t) d t \\
& =\left(\alpha_{1}+\alpha_{2}\right) \int_{0}^{\delta} x_{1}(t) d t+\int_{0}^{\delta} h_{1}(t) d t
\end{aligned}
$$

This implies that

$$
\left(M-\alpha_{1}-\alpha_{2}\right) \int_{0}^{\delta} x_{1}(t) d t \leq \delta\left\|h_{1}\right\|_{C}
$$

Since $u_{0} \in \Pi$, it follows that

$$
\left(M-\alpha_{1}-\alpha_{2}\right) \kappa \delta x_{1}(s) \leq \delta\left\|h_{1}\right\|_{C}, \quad \forall s \in \mathbb{R}
$$

This implies

$$
\left\|x_{1}\right\|_{C} \leq \frac{\left\|h_{1}\right\|_{C}}{\left(M-\alpha_{1}-\alpha_{2}\right) \kappa} .
$$

Let $R>\max \left\{\frac{\left\|h_{1}\right\|_{C}}{\left(M-\alpha_{1}-\alpha_{2}\right) \kappa}, r\right\}$. Then the condition of Lemma 3 holds.

Hence by Lemma 3, we have

$$
i\left(\Lambda, \Pi \cap \Delta_{R}, \Pi\right)=1 .
$$

Combining (3.6) and (3.7), utilizing the additivity of fixed point index, we have

$$
i\left(\Lambda, \Pi \cap\left(\Delta_{R} \backslash \bar{\Delta}_{r}\right), \Pi\right)=i\left(\Lambda, \Pi \cap \Delta_{R}, \Pi\right)-i\left(\Lambda, \Pi \cap \Delta_{r}, \Pi\right)=1 \neq 0 .
$$

Hence, $\Lambda$ has at least one fixed point in $\Pi \cap\left(\Delta_{R} \backslash \bar{\Delta}_{r}\right)$, which is a positive $\delta$-periodic solution of the second-order delayed differential equation (1.1). 


\section{Competing Interests}

None of the authors have any competing interests in the manuscript. All authors contributed equally in writing this paper. All authors read and approved the final manuscript.

\section{Acknowledgements}

The work is supported by Gansu Natural Science Foundation (No.: 1506RJYA018) and Gansu Technology Plan (No.: 17JR5RA071).

\section{References}

[1] K. Deimling, Nonlinear Functional Analysis, Springer-Verlag, New York, 1985.

[2] D. Guo and V. Lakshmikantham, Nonlinear Problems in Abstract Cones, Academic Press, New York, 1988.

[3] D. Jiang and J. Wei, Monotone method for first- and second-order periodic boundary value problems and periodic solutions of functional differential equations, Nonlinear Anal. 50(7) (2002), 885-898.

DOI: https://doi.org/10.1016/S0362-546X(01)00782-9

[4] Y. Li, Positive periodic solutions of nonlinear second-order ordinary differential equations, Acta Math. Sinica (Chin. Ser.) 45(3) (2002), 481-488.

[5] Y. Li, Positive periodic solutions of first and second-order ordinary differential equations, Chin. Ann. Math. 25(3) (2004), 413-420.

DOI: https://doi.org/10.1142/S025295990400038X

[6] Q. Li and Y. Li, Existence and multiplicity of positive periodic solutions for secondorder functional differential equations with infinite delay, Electron. J. Differ. Equations 2014 (2014), 1-14.

[7] Q. Li and Y. Li, Monotone iterative technique for second order delayed periodic problem in Banach spaces, Appl. Math. Comput. 270 (2015), 654-664.

DOI: https://doi.org/10.1016/j.amc.2015.08.070

[8] R. Ma, B. Yang and Z. Wang, Positive periodic solutions of first-order delay differential equations with impulses, Appl. Math. Comput. 219(11) (2013), 6074-6083.

DOI: https://doi.org/10.1016/j.amc.2012.12.020

[9] R. Olach, Positive periodic solutions of delay differential equations, Appl. Math. Lett. 26(12) (2013), 1141-1145.

DOI: https://doi.org/10.1016/j.aml.2013.06.009 
[10] Y. Wang, H. Lian and W. Ge, Periodic solutions for a second-order nonlinear functional differential equation, Appl. Math. Lett. 20(1) (2007), 110-115.

DOI: https://doi.org/10.1016/j.aml.2006.02.028

[11] Y. Wu, Existence nonexistence and multiplicity of periodic solutions for a kind of functional differential equations with parameter, Nonlinear Anal. 70(1) (2009), 433-443.

DOI: https://doi.org/10.1016/j.na.2007.12.011 